\title{
Ejercicio del sufragio: Factores que lo determinan en estudiantes universitarios
}

\author{
Ramón Eduardo Álvarez Torres, Rogers Eduardo Cabrera Sandoval ${ }^{1}$ \\ Rolando Ardón Ledezma ${ }^{2}$
}

\section{RESUMEN}

Las elecciones, en política, son un momento cumbre para la democracia. Siguiendo este enunciado, el propósito del estudio fue describir los factores sociodemográficos, políticos y psicológicos del estudiantado universitario, y conocer como estos determinan su ejercicio del sufragio. Siendo importante, por lo significativa que es la participación de esta población en la elección de los representantes políticos del país.

El enfoque es de tipo mixto; en lo cuantitativo se utilizaron los diseños transeccional descriptivo y correlacional; mientras que en lo cualitativo se utilizó un diseño sistemático. Se realizó tomando una muestra de 397 estudiantes de la UNAH-CU, con un nivel de confianza del $95 \%$. Se encontró que dos tercios de los universitarios votan con regularidad. La edad, el nivel académico, el interés por la política y el conocimiento de las propuestas se relaciona con la frecuencia de voto.

Sus principales motivos para ejercer el sufragio son el deber cívico, la convicción propia y el temor a que utilicen el voto. En contraste, consideran que las demás personas votan por conveniencia, y lealtad a un partido; desplazando a ellas aspectos negativos sobre la responsabilidad ante la elección de un mal candidato. De las verbalizaciones obtenidas, destaca como principal característica deseada para un candidato político, la honradez/honestidad.

Explorando la polaridad, se encontró que no existe una relación entre la ideología política expresada y la ideología política latente, lo que significa que muchos a pesar de considerarse seguidores de una postura ideológica, piensan y actúan de manera contraria a la misma.

Palabras clave: ejercicio del sufragio, estudiante universitario, interés por la política, conocimiento de propuestas, deber cívico, convicción propia, temor a que utilicen el voto, conveniencia, tradición, lealtad a un partido, polaridad.

\footnotetext{
Facultad de Ciencias Sociales, Escuela de Psicología, UNAH.

${ }^{2}$ Asesor, Facultad de Ciencias Sociales, Escuela de Psicología, UNAH. Contacto: rolandoledezma@gmail.com
} 


\section{ABSTRACT}

The elections, in politics, are a peak moment for democracy. Following this statement, the purpose of the study was to describe the demographic, political and psychological factors of university students, and know how they determine them exercise of suffrage. Being important for the significant of the participation of this population in the election of political representatives of the country.

The approach is of mixed type; in the quantitative were used the descriptive and correlational designs; while in the qualitative were used the systematic design. Was performed by taking a sample of 397 students in UNAH-CU, with a confidence level of $95 \%$. It found that two thirds of college students vote regularly. Age, academic level, interest in politics and knowledge of the proposals, are related to the frequency vote.

Their main reasons to vote are civic duty, conviction and fear to use the vote. In contrast, think that other people vote for convenience, and party loyalty; displacing them, negatives aspects about responsibility for choosing a bad candidate. Of verbalizations obtained, stands out as the main desired characteristic for a political candidate, trustworthiness / honesty.

On exploration of the polarity, it found that there is no relationship between political ideology expressed and latent political ideology, which means that despite many followers considered them, part of an ideological stance, they think and act contrary to the same.

Keywords: exercise of suffrage, university students, interest in politics, knowledge of proposals, civic duty, conviction, fear of other use the own vote, convenience, tradition, party loyalty, polarity.

\section{INTRODUCCIÓN}

Los hechos de junio de 2009 fueron un retroceso para la vida democrática del país. Al respecto, el estudio Democracia en Honduras, valores y participación política (USAID, 2011), señala que la sociedad hondureña duda de su democracia. De hecho, el apoyo a la misma, en Honduras es de apenas un $53 \%$, frente a un $61 \%$ en América Latina.

A su vez, Honduras cuenta con uno de los niveles más bajos de participación electoral en la región, siendo el cuarto país con el porcentaje más bajo de votantes con un $60.9 \%$, esto basado en las elecciones de 2009. Sin embargo, la participación 
en Honduras no puede considerarse baja, en términos absolutos, ya que casi dos tercios de las personas con derecho a votar lo hicieron (USAID, 2010).

Honduras ha pasado de cinco a nueve partidos políticos en 2013, lo que denota que la contienda electoral tomará aún mayor fuerza con el nacimiento de nuevos partidos políticos y la participación de los ciudadanos. Es por ello que sería apropiado tener un mayor conocimiento de aspectos relacionados al voto, desde en un enfoque psicológico, lo que permitirá mejorar la participación electoral y fortalecer la democracia.

Así, se tendría una mayor comprensión de los procesos subyacentes a los hechos políticos que acontecen en la vida cotidiana y se lograría una decisión electoral más apropiada. Considerando todo lo anterior, la presente investigación pretende conocer cuáles son los factores que determinan el ejercicio del sufragio en estudiantes del primer período académico del año 2013 de la UNAH-CU, ya que esta población posee diversas características y puede ofrecer aportes valiosos al desarrollo del país.

Este estudio se basa en tres modelos explicativos del porqué las personas eligen determinadas opciones frente a otras (Gonzáles y Darias, 1998). A continuación se presentan los fundamentos teóricos utilizados.

\section{Factores que determinan el voto}

En la política, a menos que se comprendan las ideas y los sentimientos de las personas que tomaron ciertas decisiones para realizar determinados actos, no se puede comprender por qué ocurrieron tales cosas. Pero, una exploración de la psicología de las personas involucradas en la actividad política provee una base única y necesaria para comprender esa actividad (Cottam, Dietz, Mastors y Preston, 2004).

Tres modelos explicativos del porqué las personas eligen determinadas opciones frente a otras

Continuando con la descripción de las características inherentes en todo proceso electoral, se deben tomar de base las investigaciones sobre el estudio de la conducta de voto, las cuales tratan de determinar qué influye sobre las orientaciones básicas de las personas hacia la política y hacia su participación en diferentes actividades políticas (Peterson, 1990). Las variables independientes comúnmente empleadas en esos estudios incluyen: factores que enfatizan las 
variables sociales, demográficas y culturales, que conforman el modelo sociológico (Lazarsfeld, Berelson y Gaudet, 1948); los que ponen un mayor énfasis en el interés y el conocimiento por la política entrarían dentro de un modelo político o racional (Downs, 1957); los que enfatizan las cogniciones, preferencias, actitudes e identificaciones que integran la personalidad, conformarían el modelo psicológico (Campbell, Gurin y Miller, 1954).

\section{Polaridad}

Otro aspecto importante a considerar dentro del modelo psicológico, es la polaridad, que se vuelve relevante en este estudio porque permite medir de forma conjunta la personalidad, los valores, la identidad y las actitudes; todas orientadas a la ideología política. Esta es considerada como una orientación básica que influye de manera decidida en el comportamiento electoral (Dalton, 1988).

Sobre este punto Tomkins (1965) dice que: "Creemos que la forma positiva o negativa en que un ser humano aprende a sentir sobre sí mismo y sobre los demás determina su postura general en el campo ideológico". Bajo esta misma línea, se propuso medir los "guiones ideológicos", es decir, las predisposiciones emocionales que subyacen a las creencias que representan la derecha, centro 0 izquierda, del continuo ideológico. Las personas con guiones ideológicos humanistas se sentirán atraídas y tendrán sentimientos positivos hacia las posiciones de izquierda en algunos de estos campos y las personas con guiones normativos serán atraídas por las ideas de derecha (Stone y Garzon, 1992).

Tomando en cuenta todo lo que se ha expuesto anteriormente, en este estudio se pretende describir los factores sociodemográficos, políticos y psicológicos en los estudiantes universitarios y conocer cómo estos factores determinan su ejercicio del sufragio.

\section{METODOLOGÍA}

\section{Diseño}

El presente estudio tiene un enfoque metodológico mixto, en el que de forma simultánea se abordan la parte cuantitativa y cualitativa; en la primera se utiliza un diseño transeccional descriptivo y correlacional. En un nivel descriptivo se establece un panorama de la conducta del voto, los factores determinantes del ejercicio del sufragio y la polaridad; y en un nivel correlacional se establece una relación entre las variables mencionadas anteriormente. 
En la segunda parte, como complemento, se utiliza un diseño sistemático para codificar las respuestas de preguntas abiertas y presentar en categorías las opiniones brindadas.

\section{Participantes}

La población de esta investigación se obtuvo del total de alumnos de la Universidad Nacional Autónoma de Honduras (UNAH), en la Ciudad Universitaria. El universo consta de 47,938 estudiantes.

Para seleccionarlos se utilizó un muestreo probabilístico estratificado, determinando una cantidad representativa según el número de estudiantes en cada facultad; para tener el tamaño de la muestra se utilizó la fórmula de Taro Yamane (1979), con un error aleatorio del $5 \%$ (0.05), alcanzando así un total de 397 estudiantes a encuestar. Las características de la muestra se describen en la tabla 1.

Tabla 1. Características de la muestra

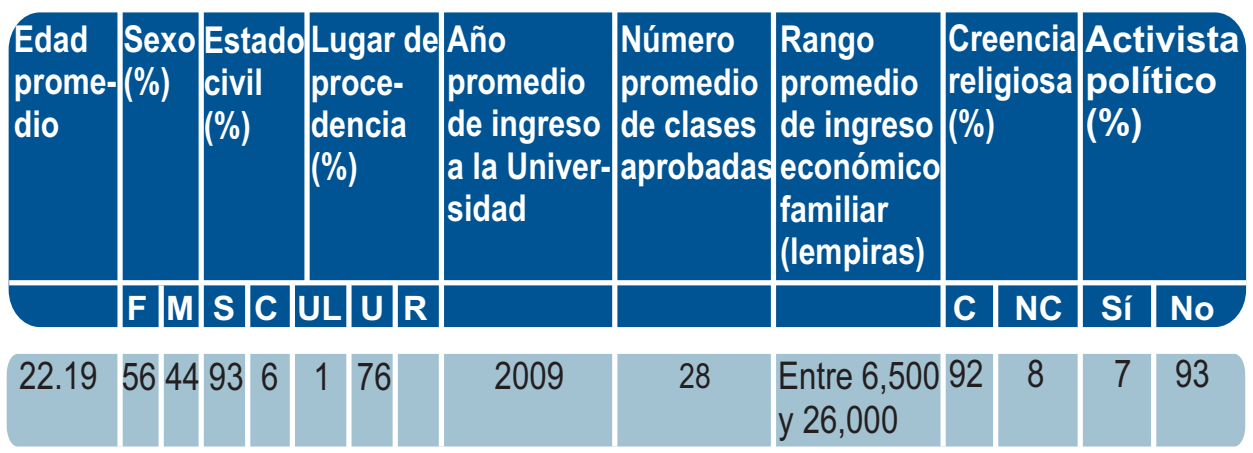

Intervenciones

Se utilizó el cuestionario de conducta de voto RREM (Álvarez, Cabrera, Montecinos y Coello, 2013), para explorar mediante diferentes variables (sociodemográficas, políticas y psicológicas) tanto la ideología política, como la conducta de voto.

El cuestionario consta de 27 preguntas, en las cuales el sujeto debe, en primer lugar, especificar sus datos de identificación (edad, sexo, estado civil, año de ingreso a la universidad, número de clases aprobadas, carrera, ingreso económico familiar, creencia religiosa, ideología política, si es activista político y si pertenece a una organización política), posteriormente debe responder a las preguntas basadas en 
aspectos generales orientados a la política, que incluye su frecuencia y tipo de voto, como también los aspectos que toma en cuenta antes de ejercer el sufragio o, en su defecto, las razones para no hacerlo, entre otros.

Igualmente se utilizó la escala de polaridad (Tomkins, 1987), la cual consta de 59 pares bipolares de ítems extraídos de distintos campos de la vida social y personal del hombre, que incluyen las creencias sobre la educación infantil, la naturaleza de la realidad, los sentimientos humanos, el cambio y la propia sensibilidad emocional de los individuos. Cada ítem polar aporta una de las cuatro respuestas posibles: humanismo, normatividad, ambos (síntesis) o ninguna (rechazo).

Contando ya con los instrumentos, se emprendió a la realización de pruebas de ensayo con estudiantes de la Carrera de Psicología, con el fin de mejorar y validar el cuestionario, solventar las dificultades que se presentaban en la aplicación de los dos instrumentos y determinar los tiempos requeridos por ambos. Posteriormente, se procedió a la aplicación de los instrumentos de recolección de datos a los 397 participantes, por medio de un muestreo aleatorio simple dentro de las diferentes facultades de la UNAH-CU.

\section{Análisis estadístico}

Para realizar los análisis correspondientes se usó el software IBM SPSS Statistics 21. Se describieron cada una de las frecuencias por ítem. A su vez, predominó la utilización de la chi cuadrado como medio para realizar los diversos análisis inferenciales y además la correlación r de Pearson para comprobar la existencia o no de una relación entre la ideología política expresada y la manifiesta.

\section{Análisis e interpretación de resultados}

Con base en los resultados se pretende hacer un análisis exhaustivo del comportamiento electoral del estudiantado universitario, detallando algunos factores que lo determinan, para luego relacionarlos entre sí. De este modo, se presentarán análisis individuales de la conducta de voto pura y de cada uno de los tres modelos explicativos del porqué las personas eligen determinadas opciones frente a otras.

En cada uno de ellos se mostrarán las estadísticas descriptivas con base a los resultados obtenidos de los instrumentos y luego se analizarán para poner a prueba las hipótesis y hacer inferencias. Al conocer los resultados individuales se harán contrastaciones entre todos para determinar las relaciones significativas entre las variables. 


\section{Conducta de voto}

El ejercicio del sufragio es el momento clave de todo proceso electoral, por ello es de vital importancia tener una panorámica general de cómo se lleva a cabo el mismo. A continuación se detalla la frecuencia con que se realiza, tanto en las elecciones generales como en las internas; en qué planillas se suele votar y cómo se hace; la variabilidad que involucra la participación en las elecciones anteriores y en las de noviembre de 2013.

\section{Análisis descriptivo}

Un $75 \%$ de los estudiantes ha votado alguna vez, mientras que un $25 \%$ nunca lo ha hecho. Dentro de las principales razones para no hacerlo, se encuentran: no tener la edad requerida, y la falta de interés y convencimiento por parte de los candidatos 0 partidos políticos. Basándose en lo anterior, la mayoría de los estudiantes han optado por formar parte de los procesos democráticos del país, sin embargo, una pequeña parte de la población muestra descontento hacia la política nacional.

En los siguientes resultados se tomará como población para el análisis el 75 \% (299) de los sujetos, ya que el $25 \%$ (98) no ha votado nunca. Para evaluar la continuidad en el voto se les pregunto a los estudiantes quiénes habían votado en todas las elecciones desde que obtuvieron su tarjeta de identidad, a lo cual respondieron de la siguiente manera: un $70 \%$ sí se ha mantenido votando, mientras que un $29 \%$ no lo ha hecho con regularidad (1 \% NS/NR). Lo que indica que en la mayoría predomina un voto estable. Asimismo, dentro de las razones que rompen la continuidad en el voto están: ningún candidato era de mi simpatía, motivos personales (viajes, enfermedad, trabajo, entre otros), baja motivación, lejanía del centro de votación y, en menor medida, por el golpe de Estado.

Para evaluar la progresión del voto se exploró la frecuencia del mismo en las tres elecciones más recientes, en donde se observa lo siguiente: en las elecciones generales de 2009 , un $64 \%$ del estudiantado podía votar, de los cuales el $74 \%$ lo hizo y un $26 \%$ se abstuvo. En las elecciones internas de 2012, un $75 \%$ de los estudiantes podía votar, de los cuales un $81 \%$ lo hizo, mientras que un $19 \%$ se abstuvo.

En contraste para las elecciones generales de 2013, un $76 \%$ manifestó que si lo haría, un $4 \%$ se abstendría y un $20 \%$ no lo sabía. Con base a lo propuesto, se observa una reducción en el porcentaje de abstinencia, lo que denota un incremento en el interés de una votación a otra. 
El estudiantado que votó en las elecciones generales de 2013, expresó que lo haría porque lo consideraba en mayor medida un deber cívico; entre otras razones se destacan el deseo de un cambio y la existencia de un buen candidato. Con menor intensidad se presentan: hacer valer su voto y ejercer ese derecho. Es importante destacar, por parte de los estudiantes, considerar el voto más como un deber que un derecho. Por otro lado, dentro de las razones que ponen en duda la participación del estudiantado, impera el considerar la existencia de malos candidatos y que tienen que analizar sus propuestas.

Una vez descrita la progresión y consistencia de los estudiantes en cuanto al voto, es importante delimitar cómo suele votar el estudiante universitario. Se encontró que un $90 \%$ selecciona a cada uno de sus candidatos, un $4 \%$ lo hace al azar, un $3 \%$ en plancha y otro $3 \%$ votan nulo. Todo lo anterior denota la importancia que se da al hecho de hacer una buena elección de los candidatos.

Bajo esta misma línea se debe dilucidar en qué planillas suele votar. Se encontró que un $97 \%$ vota por el presidente, un $88 \%$ por los alcaldes y un $84 \%$ por los diputados. En su conjunto, un $77 \%$ vota en todas las planillas y un $23 \%$ vota omitiendo ciertos cargos a elección popular; de esta manera, el voto presidencial es el que toma mayor importancia.

También es interesante saber si el estudiantado ha votado siempre por el mismo partido para conocer la variabilidad en la participación electoral. Se encontró que un $78 \%$ no siempre vota por un mismo partido y un $22 \%$ se mantiene fiel a su partido 0 candidatos. Lo que indica que la decisión del estudiantado varía en cada uno de los distintos procesos electorales.

\section{Modelo sociológico}

Desde este modelo se busca relacionar a las personas con las características demográficas individuales y grupales. Estas se toman como base para inferir diferencias orientadas a una variable específica. Los aspectos tomados en cuenta en este estudio son: edad, sexo, estado civil, nivel educativo, procedencia y estatus socioeconómico, todos ellos se contrastaron con la frecuencia de voto y la identificación por partidos políticos, debido a que en la literatura revisada se presentan antecedentes sobre estos cruces y, por ende, se buscan explorar los resultados en estudiantes universitarios.

\section{Análisis inferencial}

Los análisis se realizaron mediante el procedimiento estadístico chi cuadrado, para 
ver las diferencias entre los grupos, es decir, se pretende explorar si algunas variables sociodemográficas presentan diferencias entre grupos al contrastarlas con variables de otros modelos. En la tabla 2 se presenta un resumen de los datos obtenidos en los análisis.

Tabla 2. Datos obtenidos mediante chi cuadrado entre variables sociodemográficas y algunas de otros modelos

\begin{tabular}{|c|c|c|c|c|c|c|}
\hline $\begin{array}{l}\text { Variables } \\
\text { comparadas }\end{array}$ & Frecu & incia de & voto & $\begin{array}{l}\text { Identil } \\
\text { polític }\end{array}$ & $\begin{array}{l}\text { cación } \\
\text { os }\end{array}$ & or partidos \\
\hline & $X^{2}$ prueba & $X^{2}$ table & Significancia & $\overline{X^{2} \text { tabla }}$ & $\overline{X^{2} \text { prueb }}$ & Significancia \\
\hline Edad & 59.694 & 13.277 & $\begin{array}{l}\text { Significativo al } \\
0.01\end{array}$ & 10.134 & 14.067 & $\begin{array}{l}\text { No significativo } \\
\text { al } 0.05\end{array}$ \\
\hline Sexo & 0.275 & 6.635 & $\begin{array}{l}\text { No significativo } \\
\text { al } 0.05\end{array}$ & 9.021 & 6.635 & $\begin{array}{l}\text { Significativo al } \\
0.01\end{array}$ \\
\hline Estado civil & 0.183 & 9.219 & $\begin{array}{l}\text { No significativo } \\
\text { al } 0.05\end{array}$ & 0.555 & 9.210 & $\begin{array}{l}\text { No significativo } \\
\text { al } 0.05\end{array}$ \\
\hline Nivel educativo & 25.469 & 11.345 & $\begin{array}{l}\text { Significativo al } \\
0.01\end{array}$ & 9.024 & 16.812 & $\begin{array}{l}\text { No significativo } \\
\text { al } 0.05\end{array}$ \\
\hline Procedencia & 6.405 & 5.991 & $\begin{array}{l}\text { Significativo al } \\
0.05\end{array}$ & 3.525 & 9.210 & $\begin{array}{l}\text { No significativo } \\
\text { al } 0.05\end{array}$ \\
\hline $\begin{array}{l}\text { Estatus } \\
\text { socioeconómico }\end{array}$ & 0.304 & 11.345 & $\begin{array}{l}\text { No significativo } \\
\text { al } 0.05\end{array}$ & 3.037 & 11.345 & $\begin{array}{l}\text { No significativo } \\
\text { al } 0.05\end{array}$ \\
\hline
\end{tabular}

Se encontró que la edad y el nivel educativo son factores determinantes en el voto, en donde los estudiantes a medida que crecen y se educan tienen una mayor conciencia del valor que posee el mismo, efectuándolo mayormente. También los estudiantes que viven en entornos urbanos suelen votar más que los que viven fuera de las ciudades; esto se relaciona con lo planteado por Lipset (1969), quien menciona que las personas con mayor educación votan más que los menos educados; el citadino más que quienes habitan en espacios rurales; los que tienen entre treinta y cinco y cincuenta años votan más que los más jóvenes.

Se observa que las mujeres universitarias tienden a identificarse (por ende a brindar su voto) más por partidos conservadores, mientras que los varones lo hacen en mayor medida por partidos progresistas; esto concuerda con estudios realizados que argumentan que las mujeres parecen votar de forma más conservadora que los hombres (Avery, 1988; Ekehammar y Sidanius, 1982; González y Peñate, 1996).

Por otra parte, no se encontraron diferencias significativas en cuanto al voto según 
sexo y nivel socioeconómico; tanto hombres como mujeres, independientemente de su ingreso económico familiar, votan con una frecuencia similar. Por lo anterior, en el contexto universitario no se aplica lo planteado por Lipset (1969), quien expone que las pautas de participación política muestran un parecido sorprendente entre países como Suecia, Estados Unidos, Noruega, Finlandia y otros, en los cuales encontró que los hombres votan más que las mujeres y las personas de mayor estatus más que las de bajo. Lo cual denota que los tiempos han cambiado y que las estudiantes universitarias actualmente no se han rezagado en cuanto a ejercer su derecho de votar.

Asimismo, se observa que la edad y el estatus socioeconómico no parecen tener incidencia sobre la predilección partidista de los estudiantes universitarios, esto denota que en este entorno no hay tendencias marcadas hacia un tipo de partido en específico. Lo cual discrepa con las investigaciones llevadas a cabo con relación a las características sociodemográficas que postula el modelo sociológico, donde apuntan a que las personas de posición socioeconómica alta votan por partidos conservadores y los de posición baja por partidos progresistas (Centers, 1949; Larzarsfeld, Berelson y Gaudet, 1948; Glenn, 1973).

\section{Modelo político}

Para comprender la conducta de voto es necesario establecer relaciones con los distintos modelos que tienen influencia sobre esta, por lo tanto, es importante comenzar con la descripción de aspectos políticos o racionales, tales como el interés y conocimiento por la política y cómo estos tienen una injerencia sobre la toma de decisiones a nivel electoral. A continuación se presentan los resultados obtenidos.

\section{Análisis descriptivo}

Se comienza describiendo el interés por la política en general. Se observa que un 37 $\%$ muestra un interés moderado, un $34 \%$ poco, un $16 \%$ ninguno y un $13 \%$ bastante. En cuanto a la política a nivel nacional, los valores cambian levemente con respecto a lo anterior, así, un $40 \%$ presenta un interés moderado, un $34 \%$ poco, un $14 \%$ bastante y un $12 \%$ ninguno. Esto indica que la mayoría de los estudiantes presentan un interés por la política entre moderado y poco, tanto a nivel general como nacional.

De igual manera, se le preguntó a los estudiantes univeritarios si consideran un deber ciudadano informarse sobre la situación politica del país, a lo que 
respondieron de la siguiente manera: un $96 \%$ dijo sí y un $4 \%$ que no. De los que respondieron afirmativamente, un $62 \%$ considera necesario hacerlo siempre, un 24 $\%$ periódicamente, un $8 \%$ ocasionalmente, un $2 \%$ en época electoral y un $2 \%$ cuando sucede un evento importante. En su mayoría, se observa que desean estar informados sobre los diversos acontecimientos de la política nacional y consideran adecuado hacerlo frecuentemente.

De forma específica sobre la política nacional, se exploró el interés por conocer información sobre los candidatos politicos, a lo cual un $44 \%$ respondió moderado, un $28 \%$ bastante, un $23 \%$ poco y un $4 \%$ ninguno. Con relación al conocimiento de esta información, se les preguntó si conocen sus propuestas. Se encontró que un 56 $\%$ conocen algunas, un $28 \%$ las desconoce y solamente un $16 \%$ afirma conocerlas. Al $72 \%$ de las personas que conocen todas o algunas de las propuestas, se les preguntó qué opinión tienen de ellas y un $58 \%$ las consideran regulares, un $19 \%$ malas, un $9 \%$ muy malas, en contraste un $9 \%$ difiere de estas considerando que son buenas y un $2 \%$ que son excelentes ( $3 \%$ NS/NR). Se observa que la mayoría de las personas expresan conocer sobre las propuestas políticas y la opinión que predomina sobre ellas es regular.

Por último, continuando con el interés del estudiantado sobre la política, se preguntó si se interesan por involucrarse en aspectos concernientes a algún partido político, a lo cual respondieron así: un $82 \%$ dijo no y un $18 \%$ que sí. Se puede afirmar que en su mayoría los universitarios no participan en las actividades que realizan los partidos políticos.

\section{Análisis inferencial}

Como continuación a los datos descriptivos, se presenta el análisis específico de las variables políticas estudiadas en comparación con algunas variables de la conducta de voto y de otros modelos, para determinar su incidencia sobre el sufragio.

Existen diferencias significativas al comparar el nivel de interés con la frecuencia de voto $\left(X^{2}\right.$ prueba $=9.865, X^{2}$ tabla $\left.=7.815, p>0.05\right)$. Lo que indica que el interés sobre la política se relaciona con la conducta de voto, en consecuencia, entre más interés exista, habrá una mayor intención de voto por parte del estudiantado universitario. Además, hay diferencias significativas al comparar el conocimiento de las propuestas de los candidatos con la frecuencia de voto ( $X^{2}$ prueba $=14.257, X^{2}$ tabla $=$ $9.210, p>0.01)$. Con base en lo anterior, se puede afirmar que conocer las propuestas de los candidatos está relacionado con el ejercicio del sufragio, lo cual denota que las personas que sí conocen al menos algunas de las propuestas, tendrán una mayor intención de votar. 
Para complementar el análisis anterior, se contrastó el hecho de considerar un deber ciudadano informarse, con el hecho de conocer las propuestas de los candidatos electorales; en respuesta, se encontraron diferencias significativas $\left(X^{2}\right.$ prueba $=18.409, X^{2}$ tabla $\left.=9.210, p>0.01\right)$, por lo que se puede afirmar que a pesar de que la mayoría de los universitarios consideran valioso y necesario conocer sobre la situación política del país, pocos se comprometen con esta actividad, al punto que no llegan a conocer la mayoría de las propuestas de los candidatos a elección popular.

\section{Modelo psicológico}

Este modelo toma como premisa que el comportamiento de las personas se ve influido de gran manera por su estado psicológico interno y la interacción de este con el ambiente. Para establecer un panorama de las variables psicológicas que integran este modelo, se presenta una descripción de la percepción, la opinión, las motivaciones, las preferencias, la toma de decisiones, las influencias, las identificaciones; la pertenencia y la ideología política; enfocadas en la conducta de voto y luego relacionarlas con la polaridad. A continuación se presentan los resultados obtenidos.

\section{Análisis descriptivo}

La percepción de los estudiantes hacia los candidatos y los partidos políticos es mala en un $68 \%$, regular en un $31 \%$ y buena en un $1 \%$. Lo que indica que la forma en como el estudiantado los ven, es predominantemente negativa. Las razones que más destacan son: por corruptos, no confío en ellos, son incapaces para gobernar, velan por su propio interés y no el del país y no cumplen sus promesas. Estas frases muestran el descontento que hay popularmente con los políticos y es un ejemplo del pensamiento colectivo de la población.

Con relación a las propuestas de los candidatos electorales, las opiniones que más destacan son: regulares en un $60 \%$, malas en un $19 \%$, muy malas en un $10 \%$, buenas en un $9 \%$ y excelentes en un $2 \%$; lo que indica que en su mayoría las opiniones tienen una postura intermedia, pero en las demás hay cierta tendencia a lo negativo.

En los siguientes resultados se tomarán como población para el análisis el $75 \%$ (299 personas) de los sujetos, ya que para el $25 \%$ (98 personas) no aplican las preguntas porque no han votado nunca. Retomando los procesos electorales anteriores a 2013, se les preguntó qué los había motivado a votar, presentándoles 
múltiples opciones de las cuales debían seleccionar tres, a continuación se presentan las respuestas.

Gráfico 1. Motivos propios para votar

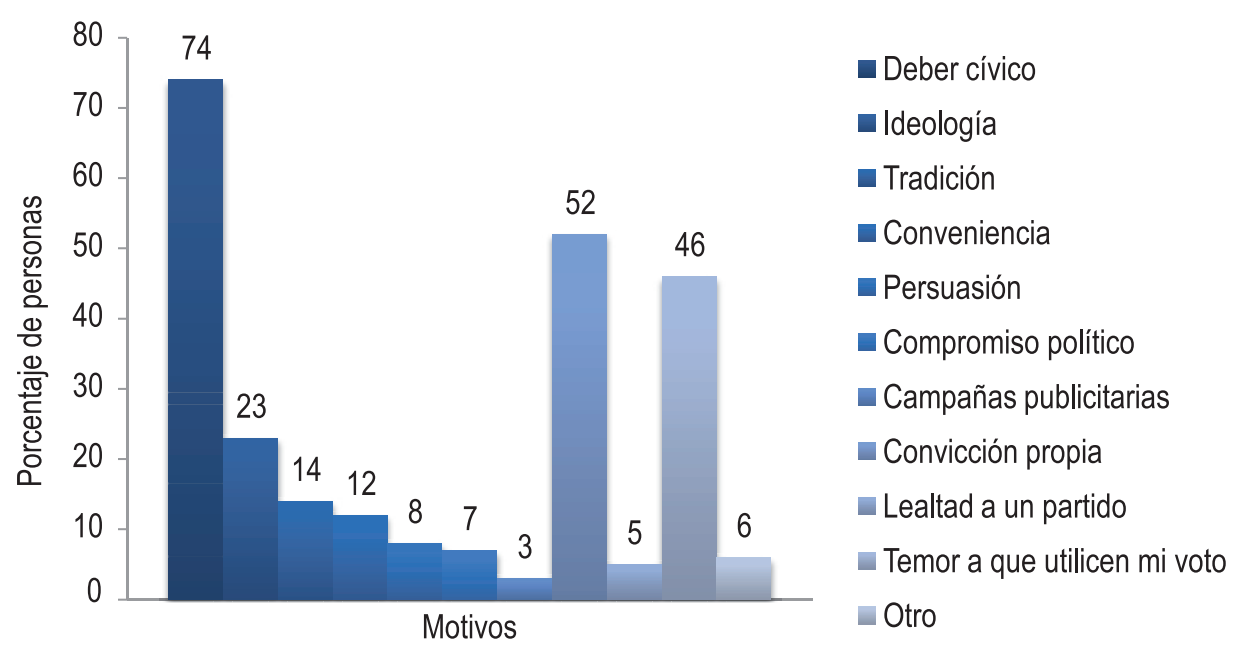

En el gráfico 1 se presenta el porcentaje de personas que escogieron cada una de las opciones, las que más destacan son: deber cívico, ya que un $74 \%$ manifestó que fue un motivo el que los llevo a votar; seguido por la convicción propia (52 \%) y luego el temor a que utilicen mi voto (46\%). Lo anterior muestra que en los universitarios hay una presencia significativa del valor patriótico que los lleva a ejercer el sufragio; luego se observa que algunas personas tienen confianza y seguridad en el acto de votar.

Por otra parte, se hace notar la presencia de la idea que las personas que no votan corren el riesgo de que ilegalmente los partidos políticos tomen su voto a conveniencia. Esto es un análisis individual de los motivos, pero al hacer un análisis de las tres opciones que más escogieron en conjunto, el trío más frecuente se constituye por los motivos mencionados anteriormente, aunque destaca otro que es: deber cívico-ideología-convicción propia.

Siempre retomando las elecciones anteriores a 2013, como razón por la que prefirieron votar por un candidato determinado, destacan las buenas propuestas (66 $\%$ ) y con una menor presencia están la simpatía (14\%), otra (10\%) y era candidato de mi partido (8\%). Dentro de la categoría otra, la opinión que más destaca es: era el 
menos peor. Lo que indica que las propuestas de los candidatos electorales tienen relevancia en la decisión de voto de las personas.

Al momento de ejercer el sufragio un $90 \%$ mantuvo la decisión de voto que tenían pensada, mientras que un $10 \%$ cambió su decisión; la razón más relevante fue que tuvieron un momento de reflexión. Esto quiere decir que la mayoría del estudiantado, una vez que ha tomado la decisión por quien votar, permanece firme en cuanto a esta.

En los siguientes resultados se vuelve a tomar en cuenta toda la muestra (397 personas), ya que las siguientes preguntas pueden ser respondidas sin que las personas hayan votado.

Como contraparte a la pregunta anterior que se refería a los motivos propios para votar, se preguntó también qué considera que motiva a otras personas a votar, bajo los mismos parámetros; esto con el fin de explorar los pensamientos que se tienen de los demás.

Gráfico 2. Motivos de otras personas para votar

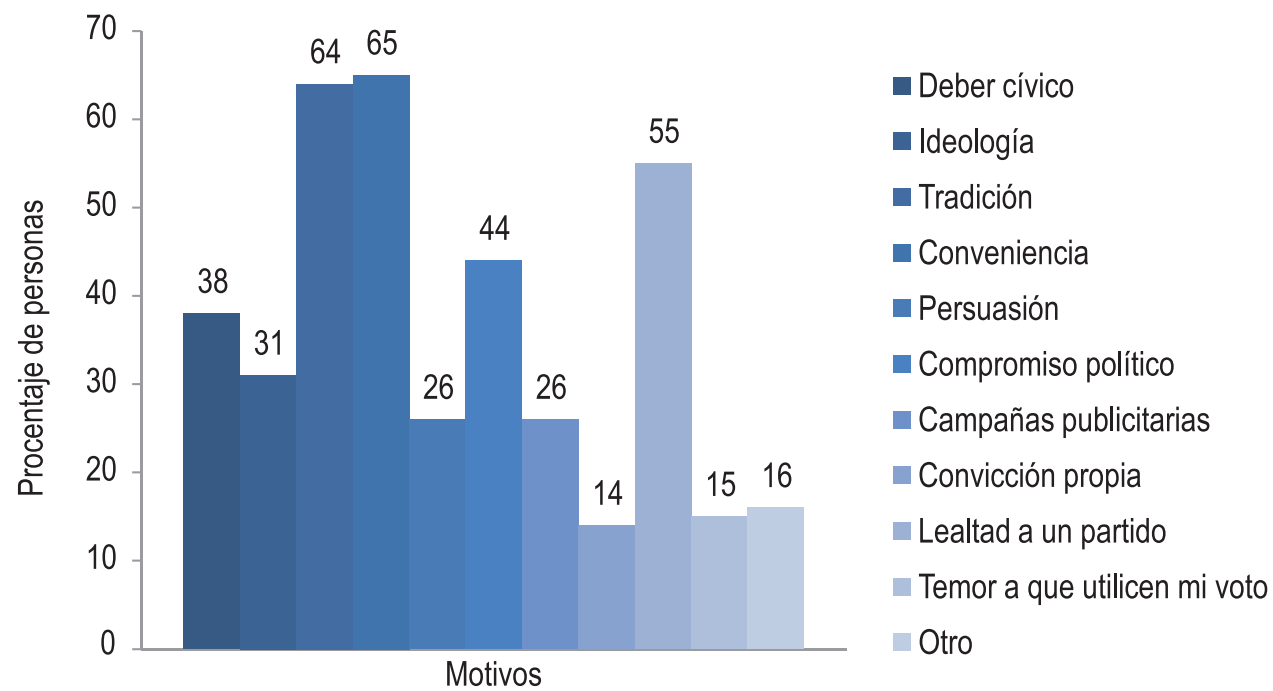

En el gráfico 2 se presenta el porcentaje de personas que escogieron cada una de las opciones, las que más destacan son: conveniencia (65\%), tradición (64 \%) y lealtad a un partido (55\%). Esto indica lo relevante que se consideran los intereses 
y beneficios individuales de las demás personas al momento de votar. Al hacer un análisis de las tres opciones en conjunto que más escogieron, el trío más frecuente se constituye por los motivos mencionados anteriormente, pero también destacan: tradición-conveniencia-campañas publicitarias y conveniencia-compromiso políticolealtad a un partido.

Anteriormente se mencionaron motivos para que ejercieran el voto, ahora se mencionarán razones que influirían para no hacerlo, aquí se dio la instrucción de marcar varias opciones a la vez. Como un análisis individual se obtuvo que un $45 \%$ dijo por falta de interés, un $32 \%$ por lejanía del centro de votación, un $30 \%$ por disponibilidad de tiempo y un $12 \%$ por otras razones. De estas últimas destacan: malos candidatos y fraude.

Luego de las elecciones internas de 2012, surgió una propuesta basada en la tecnología actual para llevar a cabo el conteo de votos, esta es la implementación del voto electrónico, se les preguntó a los participantes si consideraban que esta permitiría que pudieran ejercer su voto con plena confianza, a lo cual respondieron así: el $50 \%$ sí y el $30 \%$ no; sin embargo, el $11 \%$ no tenía conocimiento de la propuesta y para un $9 \%$ le es indiferente. En este análisis es posible señalar como una gran parte de las personas no confían en el método actual de realizar las elecciones y consideran que sería efectiva otra forma de control.

Para conocer las expectativas o deseos del electorado universitario, se les pidió escribir las tres características esenciales de un buen candidato a elección popular. La que más destacó fue honestidad/honradez, pues un $65 \%$ de los universitarios consideran que esta debe ser la característica principal; le siguen preparación académica (21\%), responsabilidad (16\%), buenas propuestas (13\%) y liderazgo (10\%). Lo anterior muestra que la honestidad debe ser un factor inherente en todo candidato a elección popular.

Como ya es sabido, las acciones del Gobierno son comentadas de diversas maneras por la población e, incluso, en los medios de comunicación se presentan noticias relacionadas. Para tener una idea de cómo repercutió la labor del Gobierno de Porfirio Lobo en la conducta de voto de los estudiantes universitarios, se preguntó si estas acciones influyen en su voto, a lo cual un $53 \%$ respondió no y un $47 \%$ sí, lo que muestra que no hay una tendencia definida. A las personas que respondieron sí, se les preguntó cómo lo hacía, las respuestas más relevantes fueron: no votaré por el partido nacional y quiero visualizar cambios, esto refleja la opinión negativa que tienen algunos estudiantes sobre las acciones de quienes dirigen al país actualmente y el deseo de un Gobierno diferente.

Continuando con aspectos que son de interés para conocer si influyen o no en el 
voto, se preguntó si las encuestas sobre quien va ganando o va a ganar influyen en su voto, a lo cual un $89 \%$ respondió no y un $10 \%$ sí; lo que indica que el electorado universitario no se ve influido por este aspecto.

Dentro de los factores psicológicos, uno muy importante por el cual se obtiene información del componente externo del ser político, es la identificación con algún partido político, que si bien es cierto se relaciona con la afiliación personal, también denota el interés y el sentido de pertenencia con los grupos sociales. Ante este aspecto, un $57 \%$ expresó no identificarse con ningún partido (a pesar de ello el $70 \%$ sí votará en las próximas elecciones) y un $43 \%$ si lo hace. Estos últimos respondieron con qué partido político lo hacen, cuyos resultados se describen a continuación: un $37 \%$ se identifica con Libre, un $23 \%$ con el PAC, un $19 \%$ con el Partido Nacional y un $14 \%$ con el Partido Liberal. Las razones más relevantes para estas identificaciones, para Libre y PAC, fueron por buenas propuestas, por un cambio y ser considerados la mejor opción, aunque para el primero intervenía también una ideología; mientras que para el Partido Nacional y Liberal únicamente destacaba la tradición, siendo más notorio en el primero.

Para continuar describiendo este modelo, se pasa a otro aporte del estudio, la polaridad o ideología política. El objetivo es presentar lo manifiesto y lo latente de esta variable, es decir, lo que se expresa verbalmente y lo que se exterioriza en lo cotidiano en varios ámbitos de la vida de forma inconsciente a través de los pensamientos y actitudes.

Para conocer la ideología política que consideran que tiene el estudiantado universitario, se solicitó que la expresaran seleccionando del 1 al 10, tomando como parámetros 1: extrema izquierda, 5: centro y 10: extrema derecha. En donde el $66 \%$ considera que tiene una ideología política neutral, el $11 \%$ de centro-izquierda, el 9 $\%$ de centro-derecha, el $8 \%$ de extrema izquierda y el $6 \%$ de extrema derecha.

Bajo esta misma línea, resulta necesario describir la ideología política latente, es decir, la que se presenta y se encuentra en las personas de manera innata, las cuales se ven reflejadas por medio de su pensamiento y acciones. Según los resultados obtenidos en la escala de polaridad de Tomkins, se encontró que el $50 \%$ del estudiantado presenta una postura humanista y el otro $50 \%$ una postura normativa, cabe mencionar que las personas humanistas se acoplan a una ideología de izquierda y las normativas a una ideología de derecha.

Con base a la distribución normal $(\dot{X}=59.707, D E=8.1615$, asimetría $=-0.121$ y curtosis $=0.465$; indica que se presenta una normalidad en la distribución), se observa que el $36 \%$ del estudiantado universitario se encuentra ubicado en una 86 
posición centro izquierda, un $36 \%$ en centro derecha, un $11 \%$ dentro de una ideología política de derecha y un $3 \%$ en una posición de extrema derecha. Por otro lado, un $11 \%$ se ubica en una posición ideológica de izquierda y solamente un $3 \%$ se encuentra en una posición de extrema izquierda; lo que indica que existe una simetría entre el pensamiento y actuar ideológico y que la población se encuentra dividida.

La distribución de porcentajes de universitarios que presentaron una postura humanista y una postura normativa, según las facultades, refleja que la Facultad de Química y Farmacia es la que más presenta una línea normativa (derecha), en la cual un $70 \%$ muestra esta condición; luego las Facultades de Ciencias Económicas e Ingeniería con un $58 \%$, se encuentran bajo esta misma línea. En contraste, la facultad más humanista es la Facultad de Ciencias Sociales, en la cual un $74 \%$ presenta esta línea de pensamiento (izquierda); le sigue la Facultad de Humanidades y Artes con un $64 \%$. El resto de las facultades presentan resultados similares en cuanto a ambas tendencias.

Se pretendía establecer si existe o no una relación entre la ideología política expresada y la latente. En niveles absolutos no existe dicha relación, por lo cual se denota que algunas personas del estudiantado universitario no tienen una ideología política acorde a lo que manifiestan ( $r=-.097$, Sig. $=0.053$ ).

\section{DISCUSIÓNY CONCLUSIONES}

El estudiantado universitario vota con mayor frecuencia con relación al resto de la población hondureña, esto denota la importancia que le dan al sistema democrático que impera en el país; pero, a pesar de ello, una cuarta parte no lo hace debido a la poca confianza que tienen en los políticos y a su falta de interés por participar en estos eventos.

En la mayoría predomina una frecuencia de voto estable dentro de las últimas tres elecciones, sin embargo, han variado los candidatos y partidos políticos por los que han votado. Al momento de ejercer el sufragio, en general seleccionan a los candidatos que consideran como la mejor opción. Hay un mayor interés por hacerlo en la casilla presidencial en comparación a otros cargos, pues es el que tomará las principales decisiones que guíen el camino del país.

Dentro del modelo sociológico, la edad y el nivel educativo tienen relación con el voto, la tendencia parece indicar que cuando las personas crecen a nivel personal y académico tienden a valorar más el ejercicio del sufragio. En contraparte, el sexo y el estatus socioeconómico no repercuten en la frecuencia de voto, lo que hace entrever 
que el estudiantado universitario sigue un patrón común e independiente a estos factores.

Otros aspectos como la edad y el estatus socioeconómico no tienen incidencia sobre la predilección política del estudiantado, contrario a lo que se podría pensar, no existen tendencias marcadas en las cuales se pueda asegurar una orientación hacia partidos progresistas o conservadores, con base a estas características.

En cuanto al modelo político, resulta evidente que las personas consideran importante conocer e involucrarse en aspectos concernientes a la política, pero pocas asumen tal responsabilidad y no se preocupan por adquirir la información necesaria que conlleve a un voto racional y consensuado basándose en las características idóneas de los candidatos.

A partir del modelo psicológico se puede entrever que los candidatos y partidos políticos no poseen una percepción favorable de parte del estudiantado. Este, en su mayoría, no confía en ellos, los consideran corruptos y que no trabajan por el país. Los estudiantes esperan que un candidato sea sincero y honesto, responsable y preparado (académicamente), para afrontar los retos que representa el país y también que sus propuestas estén acorde a las necesidades de la sociedad.

Resulta clave considerar que los motivos por los cuales el estudiantado universitario ha votado son el deber cívico (relegando el derecho que es parte fundamental y complementaria al momento de ejercer el sufragio), la convicción propia o seguridad de votar por un candidato y de participar en procesos electorales; asimismo, un motivo digno de análisis es el temor a que utilicen su voto, lo que pone de manifiesto la desconfianza que surge en el sistema utilizado actualmente para ejercer el voto. Al respecto, la mitad del estudiantado consideró el voto electrónico como esencial para tener confianza en el proceso electivo de 2013.

Por otra parte, los estudiantes atribuyen que las demás personas votan por conveniencia, tradición y lealtad a un partido, razones que no son concernientes a un voto racional. Por ello, se observa que hacen una proyección de los elementos negativos asociados a voto, librándose de esta manera de cualquier responsabilidad y culpa ante la posible selección de malos candidatos y, en consecuencia, los partidos políticos.

En cuanto a los procesos de identificación política, se observa que las nuevas opciones surgidas para estas elecciones, dominaron la predilección del estudiantado. En orden de preferencia, los partidos Libre y PAC predominaron por 
encima de los partidos tradicionales, debido a que estos eran considerados opciones de cambio y solo en el caso de Libre se sumaba la ideología.

Con relación a la polaridad, en la ideología política expresada por los votantes universitarios se encontró que dos terceras partes de la población universitaria se consideraban a sí mismas como neutrales, mientras que la parte restante se distribuye simétricamente hacia posturas de izquierda y derecha. Por otro lado, en cuanto a la ideología política latente, se observa que no hay una tendencia marcada, la mitad del estudiantado se rige bajo una postura humanista (tendencia izquierda) y la otra mitad hacia una postura normativa (tendencia derecha), lo que hace cuestionarse sobre si realmente un partido que se rige bajo una de estas líneas estaría gobernando para las mayorías.

Con respecto a lo anterior, se encontró que no existía una relación entre la ideología política expresada y la manifiesta, porque muchos, a pesar de considerarse seguidores de una línea de pensamiento, piensan y se comportan contradictoriamente a dichas posturas.

Por lo tanto, el voto a nivel universitario da pautas que indican que el voto racional y adecuado es aquel que busca candidatos comprometidos con el país y que den demostraciones de una preocupación real por medio de la cual se busca una solución a la problemática nacional, pero esta se agudiza cada vez más ante la falta de acciones concretas que permitan el desarrollo del país orientado al bienestar común.

\section{AGRADECIMIENTOS}

Ala Sociedad Estudiantil de Investigación Psicológica (SEIP-UNAH) por su apoyo y colaboración; a Rolando Ardón, jefe de la Unidad de Investigación de la Escuela de Ciencias Psicológicas y aAna Ruth Álvarez Castillo por su cooperación durante todo el proceso.

\section{BIBLIOGRAFÍA}

Campbell, A.; Gurin, G. y Miller, W.E. (1954). The voter decides. Nueva York: Harper y Row.

Cottam, M.; Dietz, B.; Mastors, E. y Preston, T. (2004). Introduction to Political Psychology. New Jersey: Lawrence Erlbaum Associates. Recuperado de: http://es.scribd.com/doc/28368391/Cottam-Et-Al-Introduction-to-Politicalpsychology

Dalton, R. J. (1988). Citizen Politics in Western Democracies. New Jersey: Chatham 
House.

Downs, A. (1957). An Economic Theory of Democracy. Nueva York: Harper y Co.

Duverger, M. (1959). An introduction to the social sciences with special reference to their methods. Nueva York: Harpery Row.

USAID. (2010). Cultura política de la democracia en Honduras, 2010. Consolidación de la democracia en las Américas en tiempos difíciles. Tegucigalpa.

Lazarsfeld, P. F.; Berelson, B. y Gaudet, H. (1948). The people's choice. Nueva York: Columbia University Press.

Peterson, S. A. (1990). Political Behavior. Londres: Sage.

Stone, W. y Garzón, A. (1992). Personalidad e ideología: la escala de polaridad. Psicología Política, 4, 65. España.

Tomkins, S. S. (1965). Affect and the psychology of knowledge. En Tomkins, S. S. e Izard, C.E. (Eds.). Affect, cognition and personality. New York: Springer. 